\title{
A New Method for Synthesis of Poly(2,6-dimethyl-1,4-phenylene oxide) and Poly(2,6-diphenyl-1,4-phenyl oxide)
}

\author{
Naser M. Al Andis \\ Chemistry Department, College of Science, King Saud University, P.O. Box 2455, Riyadh 11451, Saudi Arabia \\ Correspondence should be addressed to Naser M. Al Andis; nandis@ksu.edu.sa
}

Received 12 January 2012; Revised 6 June 2012; Accepted 6 June 2012

Academic Editor: Mohamed Farook

Copyright (C) 2013 Naser M. Al Andis. This is an open access article distributed under the Creative Commons Attribution License, which permits unrestricted use, distribution, and reproduction in any medium, provided the original work is properly cited.

\begin{abstract}
The polymerization of two monomers 2,6-dimethylphenol and 2,6-diphenylphenol was carried out by an oxidative route in the presence of $\mathrm{Cu}(\mathrm{I})$ as a catalyst and amine complex as a solvent assisted by microwave heating. The synthesized polymers were characterized by NMR spectroscopy, differential scanning calorimetry (DSC), and gel permeation chromatography (GPC). It was observed that this process of polymerization gives high yield (98 wt\%) of poly(2,6-dimethyl-1,4-phenylene oxide) (PPO) and poly(2,6-diphenyl-1,4-phenylene oxide) (PPPO) with a molar mass of $1180\left(\bar{M}_{n}\right), 1400\left(\bar{M}_{w}\right)$ and $28000\left(\bar{M}_{n}\right), 46500\left(\bar{M}_{w}\right) \mathrm{gm} / \mathrm{mol}$, respectively. A negligible amount of diphenoquinone was also observed and its dispersity was rather moderate, 1.17 and 1.68 , respectively.
\end{abstract}

\section{Introduction}

Microwave-assisted reaction was introduced in 1986 [1]. Comparing with conventional heating, it requires lesser time with higher yield. It was attributed to dielectric heating [2] and to specify the microwave effect by others [3]. However, the main advantage of microwave reaction is to maintain a uniform temperature throughout the reaction period, as a result of internal heating by irradiation [4].

An atom transfer radical polymerization (ATRP) was introduced in 1995, which allows an efficient polymerization with well-defined molar masses and narrow dispersity of polymers produced. Microwave-assisted ATRP has been successfully used for polymerization of vinylic or acrylic monomers such as styrene [5-7], methyl methacrylate [6-8], vinyl acetate, acrylonitrile, and $n$-octyl acrylate [9]. In ATRP, an organic halide (RX) is used to initiate a transition-metal compound in its lower oxidation state along with an electrondonating compound for catalyzing polymerization [10].

An oxidative polymerization of 2,6-dimethylphenol was discovered in $1956[11,12]$, leading to the formation of poly(2,6-dimethyl-1,4-phenylene oxide) (PPO). Polymerization occurred at room temperature using an amine complex of copper (I) salt as a catalyst in the presence of oxygen (Scheme 1). A high molar mass linear PPO was formed along with a small amount of diphenoquinone [13]. In this investigation it was observed that the length of the polymer chain depended on the amine type used, its molar ratio, and polarity of the solvent. According to Hasy works [14] in a similar reaction, it was noted that the diphenoquinone became its major product at a high temperature.

In this work PPO and PPPO were synthesized for the first time by oxidative polymerization using the $\mathrm{Cu}(\mathrm{I})$ in amine complex as catalyst, heating by microwave.

\section{Experimental}

2.1. Materials. The N,N,N,N,tetramethylethylenediamine (TMEDA), 2,6-dimethylphenol (DMP), and 2,6diphenylphenol were purchased from Aldrich Chemical, Germany. Benzyl chloride (BC) and dimethylformamide (DMF) were obtained from $\mathrm{BDH}$, England, acetonitrile (ACN) from $\mathrm{M} \& \mathrm{~B}$ England, and copper(I) chloride $(\mathrm{CuCl})$ from Riedel-Dettaen AG, Germany. All chemicals were used as received. The reactions were performed in a CEM Discover microwave instrument, Matthews, NC, USA.

2.2. Polymerization. In a typical run, $0.2 \mathrm{gm}(2 \mathrm{mmol})$ of $\mathrm{CuCl}$ was added to $5 \mathrm{~mL}$ of $\mathrm{ACN}$ into a single-necked 

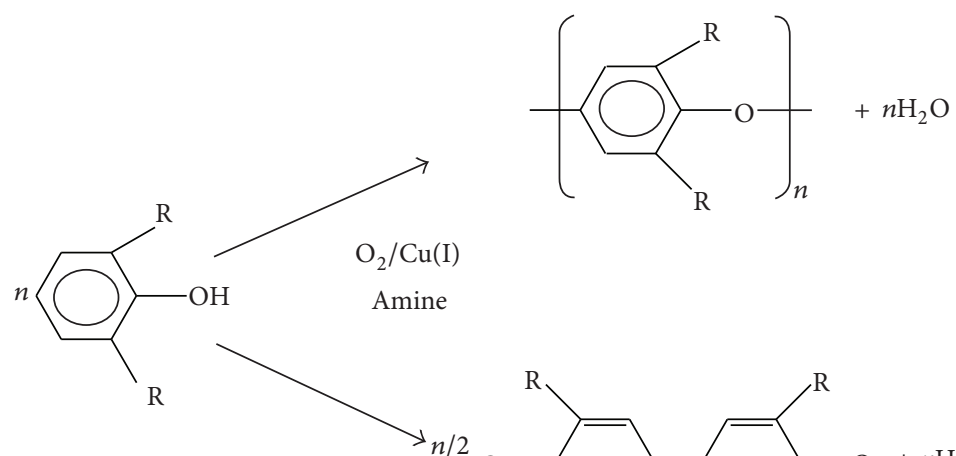

$\mathrm{R}=\mathrm{CH}_{3}$

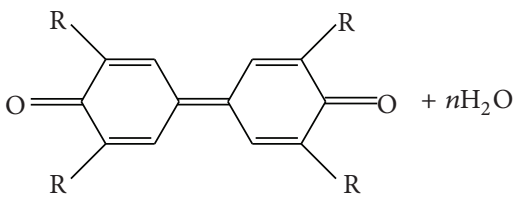

Scheme 1: Polymerization reaction of 2,6-phenylphenol by oxidation using the $\mathrm{CuCl}$ in amine.

$100 \mathrm{~mL}$ round bottom flask at room temperature. Then, $0.5 \mathrm{gm}(4.3 \mathrm{mmol})$ of TMEDA and $0.03 \mathrm{gm}(0.27 \mathrm{mmol})$ of BC were added simultaneously. The mixture was sonicated for 2 minutes at room temperature and transferred to a Pyrex tube of $100 \mathrm{~mL}$. The monomer was added to the reaction mixture after one minute. The tube was fitted with reflux and transferred to the microwave. The reaction was performed at 100 Watt for $3 \mathrm{~min}$ then at 300 Watt for $6 \mathrm{~min}$. The polymer was cooled, treated 10 times with $10 \mathrm{~mL}$ of $\mathrm{HCl} /$ methanol $(10 \mathrm{wt} \%)$, and then isolated in excess methanol. The polymers synthesized were purified by repeated dissolution in THF and precipitation in $n$-heptane, then isolated by filtration, and dried to a constant weight at $40^{\circ} \mathrm{C}$ in a vacuum oven for 5 days. Contrarily to PPO which has a white yellow color, the PPPO was light red.

2.3. Characterization. ${ }^{13} \mathrm{C}$ and ${ }^{1} \mathrm{H}$ NMR spectra of the $\mathrm{PPO}$ and PPPO in deuterated chloroform were recorded with a JEOL ECLIPSE 400 spectrometer, using tetramethylsilane (TMS) as an internal standard. The glass transition temperature $(\mathrm{Tg})$ of PPO and PPPO was measured with a DSC (Shimadzu DSC 60), previously calibrated with indium. The samples were heated from 30 to $240^{\circ} \mathrm{C}$ at a heating rate of $20^{\circ} \mathrm{C} \mathrm{min}^{-1}$. The dispersity of PPO and PPPO was determined at $30^{\circ} \mathrm{C}$ with a Viscotek Complete Tetra Detector Platform and GPCMax front-end chromatography setup equipped with 2 $\times 1$-MBMMW column by using standard polystyrene and tetrahydrofuran (THF) as a solvent.

\section{Results and Discussion}

A viscous solution appeared immediately after adding the monomer to the reaction mixture and viscosity was increased during the reaction. Under the same condition, a maximum of 98 wt \% conversion was obtained for PPO and PPPO after 3 min of the reaction time with a negligible amount of diphenoquinone. From the results of the molar mass of these two polymers, it was observed that the PPPO was relatively more active than PPO. The structures of the two polymers were confirmed by ${ }^{1} \mathrm{H}$ and ${ }^{13} \mathrm{C}$ NMR techniques.

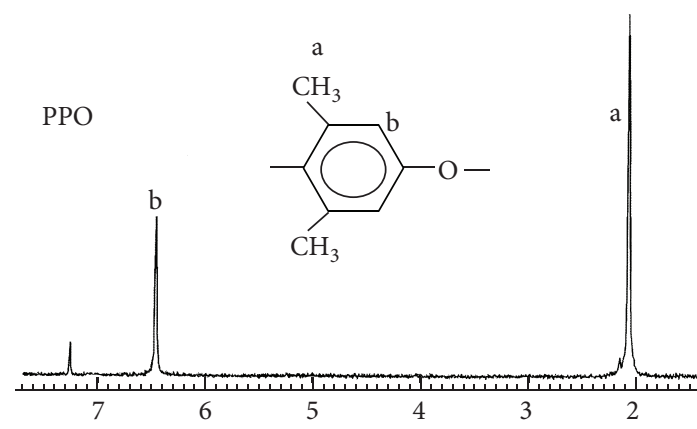

(a)

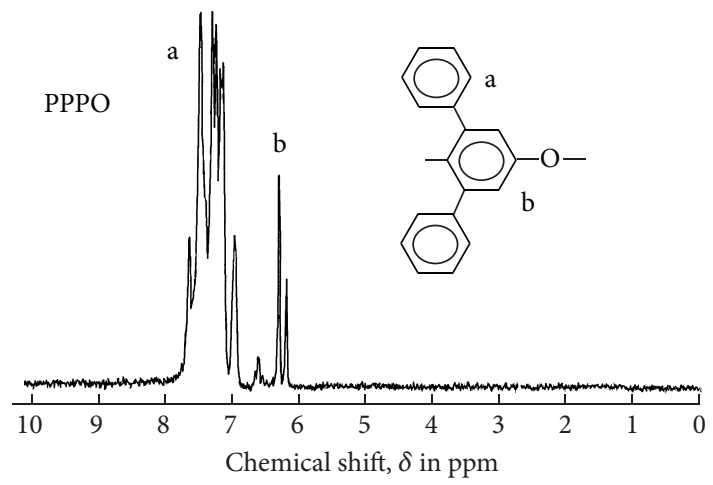

(b)

Figure 1: ${ }^{1} \mathrm{H}$ NMR spectra of PPO and PPPO.

The ${ }^{1} \mathrm{H}$ NMR spectra of PPO and PPPO are illustrated in Figure 1. It shows two sets of peaks. In the spectra of $\mathrm{PPO}$, a triplet centered at $2.11 \mathrm{ppm}$ was observed which can be assigned to the methyl protons, and one multiplet centered at $6.46 \mathrm{ppm}$ was also seen, which can be attributed to the aromatic protons. The spectra of PPPO in Figure 1 show the presence of one large multiplet between 7.04 and $7.70 \mathrm{ppm}$ assigned to 10 protons of the two substituted phenyl and another between 6.87 and $7.04 \mathrm{ppm}$ attributed to the 2 protons of the phenylene of the backbone. The absence of any peak between 4 and 5 ppm clearly indicates the disappearance 


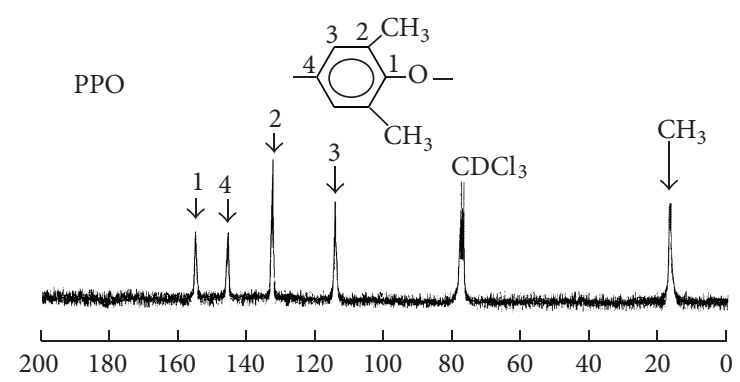

(a)

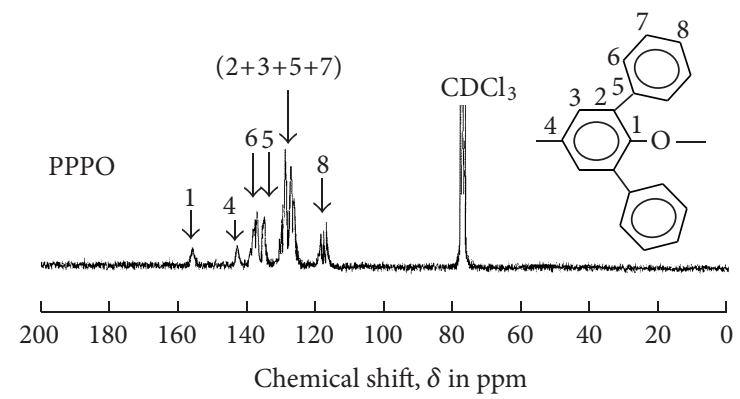

(b)

FIGURE $2:{ }^{13} \mathrm{C}$ NMR spectra of PPO and PPPO.

of hydroxyl groups, which supports the formation of the polymer.

The ${ }^{13} \mathrm{C}$ NMR spectra of PPO and PPPO are gathered in Figure 2. The spectra of PPO show a peak at $16.88 \mathrm{ppm}$ that can be assigned to the methyl carbons. Furthermore there are four sets of carbon peaks attributed to the phenyl at 154.62, $145.50,134.2$, and $114.59 \mathrm{ppm}$ assigned to the carbons $\mathrm{C}_{1}$, $\mathrm{C}_{4}$, the symmetric lateral $\mathrm{C}_{2}+\mathrm{C}_{6}$ and $\mathrm{C}_{3}+\mathrm{C}_{5}$, respectively. Concerning the PPPO, the presence of one large multiplet between 128 and $130 \mathrm{ppm}$ was assigned to the 12 carbons $\left(\mathrm{C}_{2}, \mathrm{C}_{3}, \mathrm{C}_{5}\right.$, and $\left.\mathrm{C}_{7}\right)$. The peaks at $156.2,143,136.2$, and 137.8 ppm were attributed to $\mathrm{C}_{1}, \mathrm{C}_{4}, \mathrm{C}_{6}$, and $\mathrm{C}_{5}$, respectively. The multiplet between 116.5 and $118.2 \mathrm{ppm}$ was assigned to the two carbons $\mathrm{C}_{8}$. The average molar mass, $\overline{M_{n}}$ and $\overline{M_{w}}$ of the isolated PPO are 1180 and $1400 \mathrm{gm} / \mathrm{mol}$, respectively, and those of PPPO are 28000 and $46500 \mathrm{gm} / \mathrm{mol}$, respectively, as shown in the Chromatograms of Figure 3. Their dispersity is rather moderate 1.17 and 1.68 respectively. As can be noticed, PPPO is unexpectedly obtained with the highest molar mass; PPO has two methyl groups and would rather polymerize to a lower molar mass with a tendency towards a monodisperse polymer. The DSC thermograms of PPO and PPPO plotted together in Figure 4 show a $\mathrm{Tg}$ at $210^{\circ} \mathrm{C}$ and $90^{\circ} \mathrm{C}$, respectively.

\section{Conclusion}

From these results, it can be concluded that the polymerization of 2,6-dimethyl-phenol and 2,6-diphenylphenol assisted by microwave led to the formation of poly(2,6-dimethyl1,4-phenylene oxide) and poly(2,6-diphenyl-1,4-phenylene oxide), respectively. Heating by the microwave technique

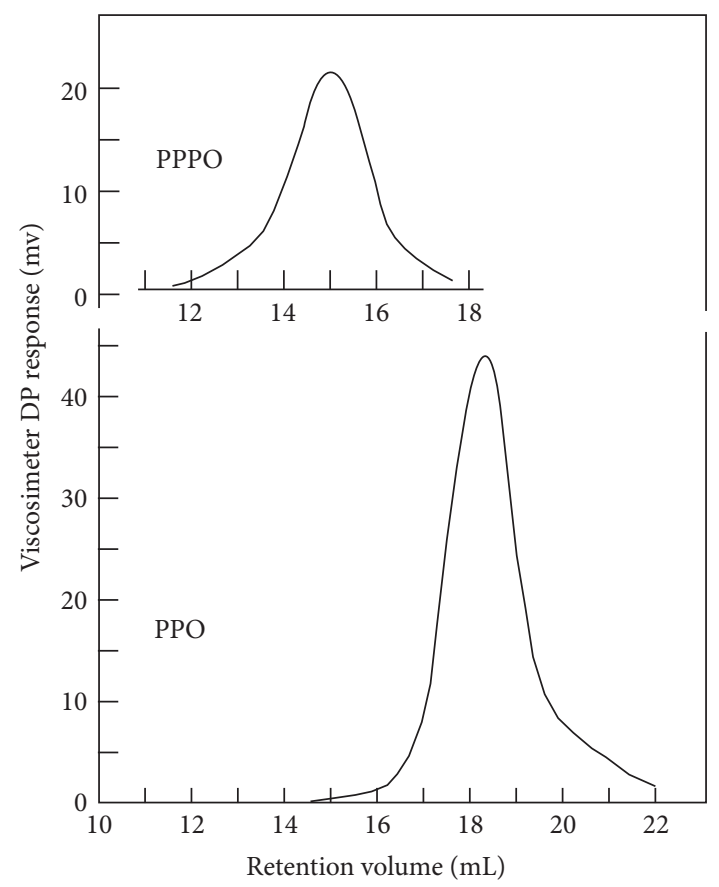

FIgURE 3: GPC chromatogram of PPO and PPPO.

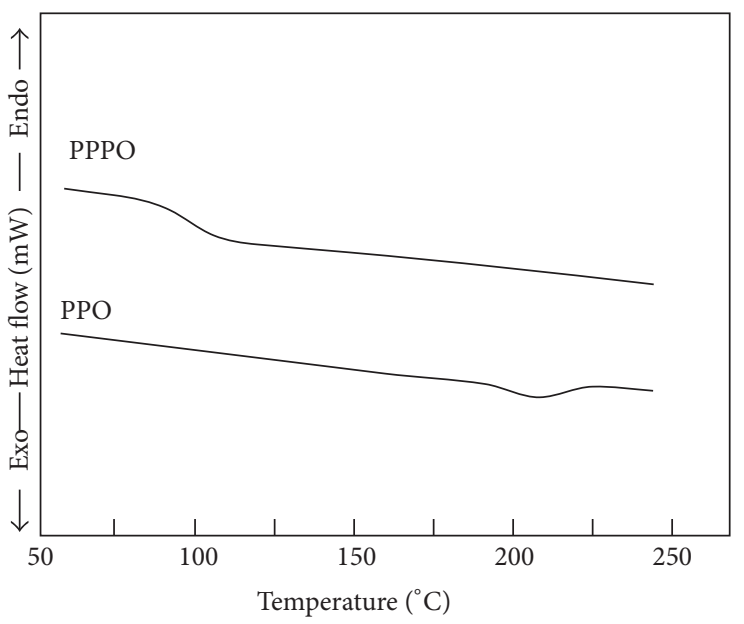

FIGURE 4: DSC thermograms of PPO and PPPO.

resulted in a high conversion to polymers after only $3 \mathrm{~min}$ and allowed obtaining moderate molar masses. From these data it was also noted that the poly(2.6-diphenyl-1,4-phenylene oxide) was relatively more active than poly (2,6-dimethyl-1,4phenylene oxide) due to the $R=\varphi$ substitute group, which increases the radical stability by resonance effect.

\section{Acknowledgment}

The author thanks King Saud University, Deanship of Scientific Research, College of Science, Research Center for the financial support under Project no. Chem/2006/15. 


\section{References}

[1] P. Lidström, J. Tierney, B. Wathey, and J. Westman, “Microwave assisted organic synthesis-a review," Tetrahedron, vol. 57, no. 45, pp. 9225-9283, 2001.

[2] C. Gabriel, S. Gabriel, E. H. Grant, E. H. Grant, B. S. J. Halstead, and D. M. P. Mingos, Chemical Society Reviews, vol. 27, pp. 213-224, 1998.

[3] R. N. Gedye and J. B. Wei, Canadian Journal of Chemistry, vol. 76, no. 5, pp. 525-532, 1998.

[4] S. Chatterjee, T. Basak, and S. K. Das, "Microwave driven convection in a rotating cylindrical cavity: a numerical study," Journal of Food Engineering, vol. 79, no. 4, pp. 1269-1279, 2007.

[5] Z. Cheng, X. Zhu, N. Zhou, J. Zhu, and Z. Zhang, "Atom transfer radical polymerization of styrene under pulsed microwave irradiation," Radiation Physics and Chemistry, vol. 72, no. 6, pp. 695-701, 2005.

[6] H. Li, S. Chen, X. Zhang, Y. Lu, and Y. Hu, "Study on the polymerizations of methyl methacrylate and styrene initiated with chlorotrimethylsilane and $\mathrm{CuCl} / \mathrm{N}, \mathrm{N}, \mathrm{N}^{\prime}, \mathrm{N}^{\prime \prime}, \mathrm{N}^{\prime \prime}$ - pentamethyldiethyltriamine," European Polymer Journal, vol. 41, no. 12, pp. 2874-2879, 2005.

[7] A. F. Porto, B. L. Sadicoff, M. C. V. Amorim, and M. C. S. De Mattos, "Microwave-assisted free radical bulk-polyaddition reactions in a domestic microwave oven," Polymer Testing, vol. 21, no. 2, pp. 145-148, 2002.

[8] Z. Chen, M. Zhu, M. Chem, J. Chen, and L. Zang, "Atom transfer radical polymerization of methyl methacrylate with low concentration of initiating system under microwave irradiation," Polymer, vol. 44, no. 8, pp. 2243-2247, 2003.

[9] W. Xu, X. Zhu, Z. Cheng, G. Chen, and J. Lu, "Atom transfer radical polymerization of $n$-octyl acrylate under microwave irradiation," European Polymer Journal, vol. 39, no. 7, pp. 1349-1353, 2003.

[10] M. K. Georges, R. P. N. Veregin, P. M. Kazmaier, and G. K. Hamer, "Narrow molecular weight resins by a free-radical polymerization process," Macromolecules, vol. 26, no. 11, pp. 2987-2988, 1993.

[11] A. S. Hay, H. S. Blanchard, G. F. Endres, and J. W. Eustance, "Polymerization by oxidative coupling," Journal of the American Chemical Society, vol. 81, no. 23, pp. 6335-6336, 1959.

[12] A. S. Hay, U.S. Patent, 3306874, 1967.

[13] A. S. Hay, "Polymerization by oxidative coupling: discovery and commercialization of PPO and Noryl resins," Journal of Polymer Science, Part A, vol. 36, no. 4, pp. 505-517, 1998.

[14] A. S. Hay, U.S. Patent, 3306875, 1967. 

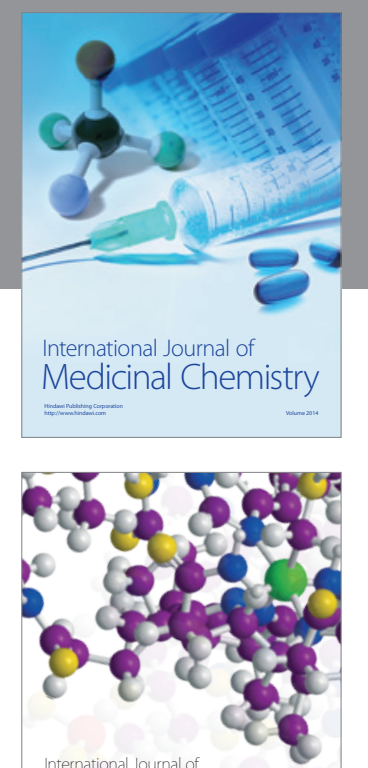

\section{Carbohydrate} Chemistry

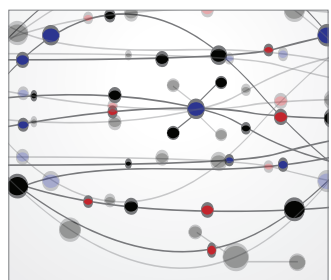

The Scientific World Journal
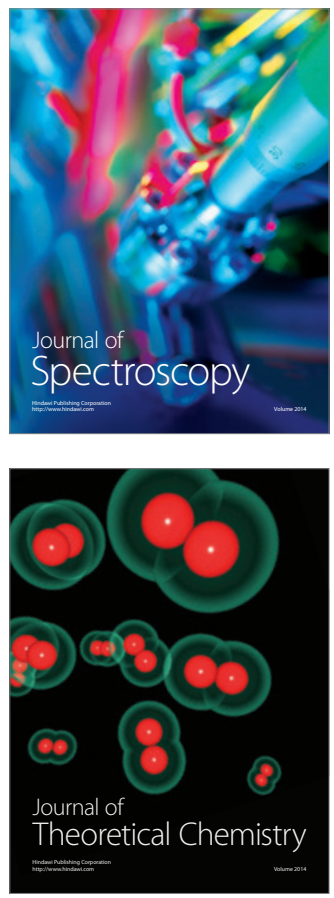
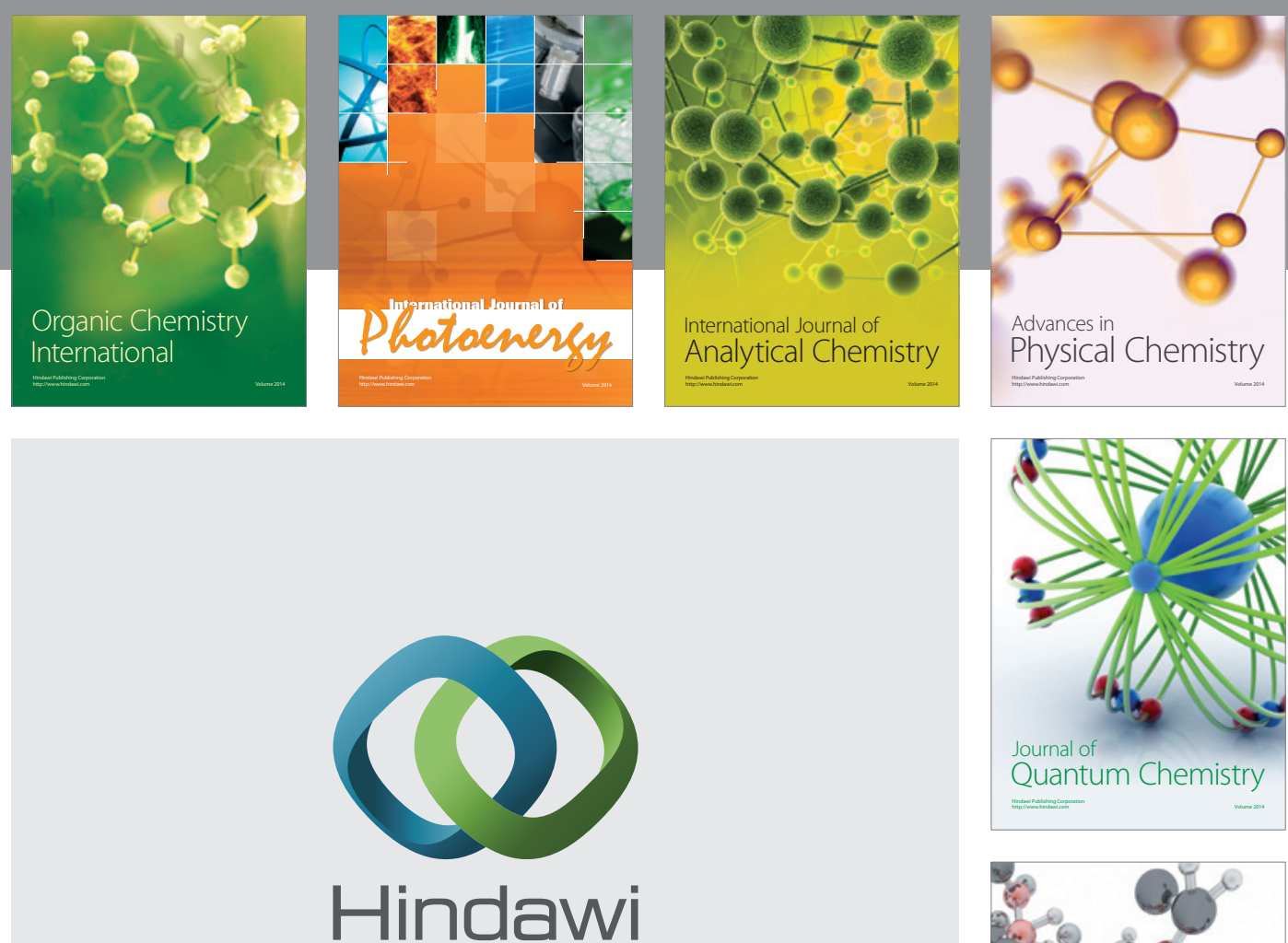

Submit your manuscripts at

http://www.hindawi.com

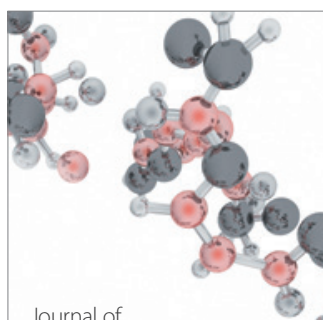

Analytical Methods

in Chemistry

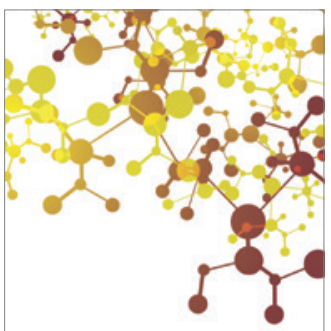

Journal of

Applied Chemistry

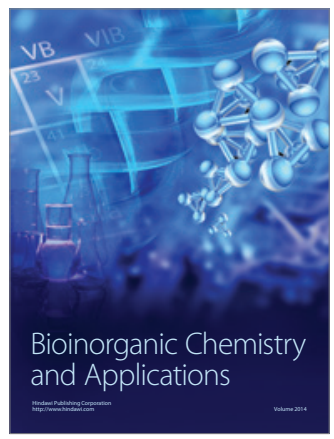

Inorganic Chemistry
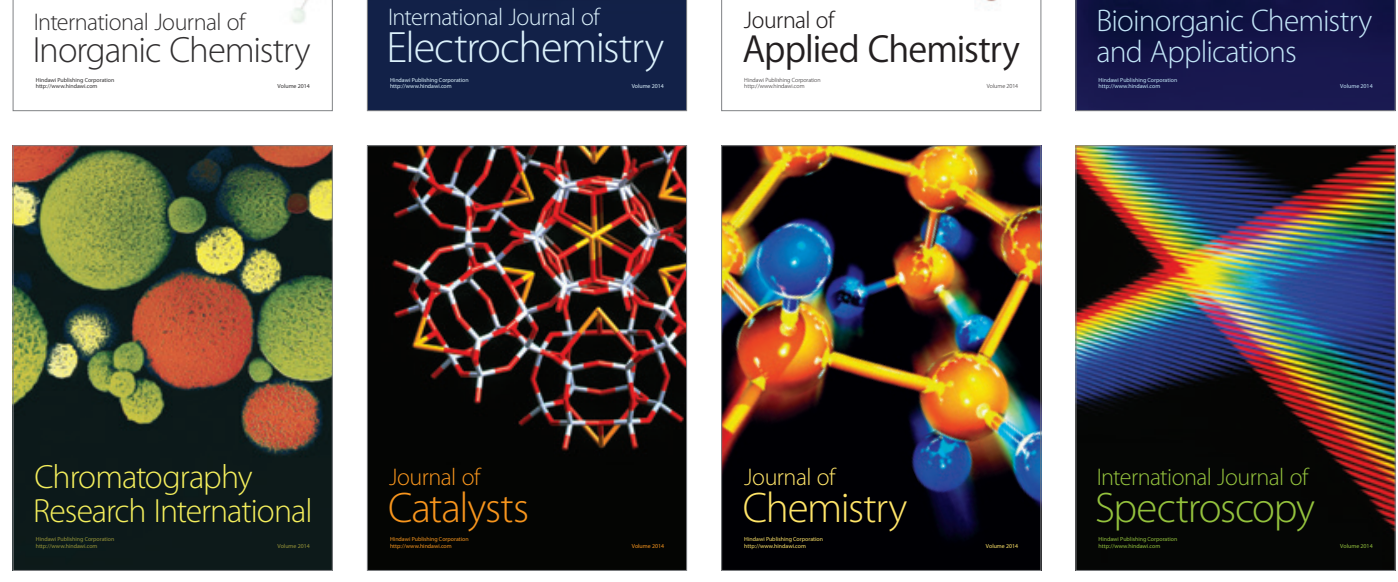